\title{
Speed Posters: An Alternative to Traditional Poster and Podium Sessions
}

Lisa D. Wagnes, MSN, RN

A novel strategy was used for some of the poster presentations for the 2015 annual Professional Nurse Educators Group (PNEG) conference. The conference planners had observed the use of a "speed poster" session at a local conference and explored its use at this conference in order to facilitate increased engagement with poster presenters and to offer variety in the type of concurrent sessions available to attendees. According to Rowe \& Ilic (2015), the second and third most frequently selected 'innovations' recommended for improvement of poster sessions by their respondents were to better organize them, specifically related to challenges in reviewing vast numbers of posters ( $56.7 \%)$, and to include a short presentation option (48.6\%) for poster presenters. The speed poster session approach used addresses both of these suggestions. The most selected innovation in the study, inclusion of the poster and a short paper in an online format, was not utilized due to resource limitations but could be considered by other conference hosts (Rowe \& Ilic, 2015).

Speed posters were chosen for several reasons. They allow attendees an opportunity to learn about a variety of topics in a short period of time, and allow presenters the opportunity to engage with people who might not have otherwise visited their poster. Some presenters also may feel more comfortable with a small group presentation versus a podium presentation. In addition, the small group discussion can provide valuable dialogue, and provoke additional questions and discussion. One factor that must be considered is accommodating for attendees who may have difficulty standing for the full session and/or moving about the room.

\section{Speed Poster Format}

This is the author's manuscript of the article published in final edited form as: Wagnes, L. D. (2016). Speed Posters: An Alternative to Traditional Poster and Podium Sessions. The Journal of Continuing Education in Nursing, 47(8), 344-346. https://doi.org/10.3928/00220124-20160715-02 
The concept of a "speed poster" is not consistently defined. In general, speed poster sessions allow presenters to briefly showcase their poster and provide attendees with snippets of the content as well as the opportunity for further discussion.

For this conference, eleven posters (of twelve accepted) were presented in two concurrent sessions, organized by interest group. Information about time and format was provided during the abstract collection period and sent to presenters prior to the conference. A rolling slide show before and after the keynote session on the day of the speed poster sessions included a slide explaining the format so attendees could better understand what to expect if they participated. During the session, attendees clustered evenly in front of each poster and each presenter was given five to seven minutes to present their poster and two minutes for Q\&A. A bell was rung and the groups moved to the next poster. The rotation schedule allowed all the attendees to hear about all the posters.

\section{Participant Feedback}

Speed poster session evaluations reflected a high level of perceived effectiveness, with mean ratings of 1.37 and 1.35 ( 1 = very effective $-5=$ very ineffective). Open ended comments showed that participants enjoyed the format, and felt it was both fun and engaging. Multiple comments reflected challenges with lack of time and spacing of posters. One respondent felt that $s /$ he did not gain any more information than at a traditional poster session.

\section{Presenter Feedback}

Thirteen responses were received from a post-conference survey sent to the nineteen presenters. Presenters were very positive about the session and format. Reported benefits included the captive audience, the ability to tell their story and engage in small group dialogue, participants' questions triggering other questions, the fast pace, feeling that it was better for participants to hear about the content than just read it, and the ability to learn about topics they might not explore on their 
own. Drawbacks described included needing to present in a very short amount of time and talk quickly, having to repeat the presentation five or six times, the noise level, needing adequate space between posters, and the problem that some participants wanted to skip a poster. Feedback about the instructions provided prior to and during the session indicated that while most respondents felt prepared, others commented that clearer expectations could be communicated prior to the session, presenters should be encouraged to bring a handout, and it should be clear that questions should be held until the end of the presentation. Anecdotally, the staff at the conference also noted that it is important to have a loud enough signal to move the session along, and that the expectation that all groups move together in the time period should be communicated clearly at the start of the session. A few attendees asked about the possibility of having the speed posters available for viewing in the general poster session.

\section{Conclusion}

The conference planners felt that the speed poster sessions were effective, engaging, and provided attendees with a new format for an interactive session. Of the 13 presenters who responded to the survey, 12 (92\%) responded that they were very likely (8) or likely (4) to submit an abstract for a speed poster presentation again, if given the opportunity. After reviewing the feedback and observations, a list of recommendations for planning speed posters was developed, as seen in Figure 1. The planning committee recommends use of this approach at conferences as space and resources permit. Speed posters can easily be adapted to larger or smaller conferences. 
Figure 1

\section{Recommendations for Speed Poster Sessions:}

\section{Physical Space and Timing}

- Ensure that there is ample space between the posters to allow people to cluster around the presenter and to minimize crossover noise.

- Provide chairs for those who may not be able to stand for extended periods.

- Consider limiting the number of attendees in the session to decrease crowding and allow for ample discussion, depending on the space available.

- Consider scheduling the speed poster breakouts during a general poster session if possible.

- Cluster related topics or audiences together in sessions to target specific groups.

- Allow ample time for the session based upon number of posters accepted. At least 8-10 minutes per poster, including Q\&A time, is recommended.

- Consider allowing posters to be displayed during general poster session and allow presenters to opt in or out of the session.

- Consider the option of providing online poster images and descriptions to allow for a wider audience if desired.

\section{Session Organization}

- Orient attendees about expectations for speed poster sessions at start of conference; for example, in the program, via slides or announcements during a general session, or on the conference website in writing or video.

- Provide moderator with clear instructions to read to presenters and attendees at the start of session, including expectation that presentations will end and all groups will move at appropriate time and that additional discussion can occur at the end of the session.

- Utilize a signal (bell, etc.) that is loud enough for all to hear.

- Provide water for presenters due to requirement of speaking quickly and presenting repeatedly.

\section{Instructions for Presenters}

- Encourage presenters to bring a handout or fact sheet to help with questions and contact information

- Provide clear communication prior to the conference, including: exact number of minutes for presenting and for Q\&A, number of times they will be asked to present, and an explanation of how session will be moderated and moved along.

- Ask presenters if they will need seating or other accommodations.

\section{Reference}

Rowe, N. \& Ilic, D. (2015). Rethinking poster presentations at large-scale scientific meetings- is it time for the format to evolve? FEBS Journal, 282, 3661-3668. doi: 10.1111/febs.13383 